\title{
Biaxial Extensional Characteristics of Drag-Reducing Surfactant Solution
}

\author{
Hong-Phuc NGUYeN*, Kiyotaka IshiHARA ${ }^{* *}$, Hiroshi SUZUKI*, Yasuo KAWAGUCHI ${ }^{* * *}$, and Hiromoto UsuI ${ }^{* * * *}$ \\ * Graduate School of Science and Technology, Kobe University, Kobe 657-8501, Japan \\ ${ }^{* *}$ Nippon Paint Co., Ltd., Neyagawa 572-8501, Japan \\ ${ }^{* * *}$ National Institute of Advanced Industrial Science and Technology, Tsukuba 305-8564, Japan \\ **** Department of Chemical Science and Engineering, Kobe University, Kobe 657-8501, Japan
}

(Received : October 6, 2004)

\begin{abstract}
The biaxial extensional characteristics of a cationic surfactant solution system showing an effective drag reduction in pipe flow have been studied by using two opposed nozzle devices. Aqueous solutions of cationic surfactant, oleylbishydroxyethylmethylammonium chloride, at the concentrations varied from 250 to 1,000 ppm with sodium salicylate, which ionized to counter-ion in an aqueous solution, of which molar ratio to surfactant was set at 1.5 , were tested. Especially, time characteristics of extensional stress were focused in wide ranges of surfactant concentration and extensional rate. From the results, the apparent extensional viscosity is found to depend on the extensional rate and on the surfactant concentration. For the extensional rate dependency, the extensional viscosity behavior is classified into four regions. This characteristic is considered to be caused by the formation and the deformation of the surfactant rodlike micellar network structure. In each region, the build-up and relaxation times are not strongly affected by the concentration of the surfactant. On the other hand, the build-up time decreases with a gradient of -1 in a double logarithm plot to the extensional rate, while the relaxation time decreases weakly with the extensional rate. The former fact indicates the micellar network formation occurs due to the collision of surfactant micelles. It is also found that two relaxation processes exist for the extensional flow cases. The shorter or the longer relaxation time takes almost the same value as each value corresponding to that for the shear flow release condition. This indicates the network structure size formed in the biaxial extensional flow takes almost the same as that in the shear flow.

Key Words: Surfactant rod-like micelle / Drag reduction / Extensional viscosity / Biaxial Extensional Flow / Build-up time
\end{abstract}

\section{INTRODUCTION}

Some aqueous surfactant solutions cause a very effective drag reduction phenomenon in turbulent flow even at very dilute concentration ${ }^{1-4)}$ as some polymer aqueous solutions ${ }^{5,6,8)}$. These aqueous solutions can reduce about $80 \%$ of the drag in turbulent flow in a straight pipe flow. So, many researchers have investigated characteristics of such a dilute surfactant solution. However, the mechanism of drag reduction occurrence has not been clarified yet because the rheological characteristics of dilute surfactant solution show complicated behaviors.

Drag-reducing surfactants form rod-like micelle in an aqueous solution. Under shear addition condition, these micellar structures form a network that is expected as a cause of the drag reduction occurrence. This micellar network adds visco-elasticity to the fluid. Some researchers pointed out that such visco-elasticity is a main cause of drag-reduction phenomena occurrence. However, Lu et al. ${ }^{7)}$ reported that a surfactant solution showing no visco-elasticity causes effective drag reduction in a pipe. They pointed out the main cause for drag reduction occurrence is not the fluid visco-elasticity but high extensional viscosity. On polymer drag reduction, high extensional viscosity was also reported to be a main cause of drag reduction occurrence ${ }^{8)}$. According to them, high extensional viscosity is considered to cause an addition of resistant against vortex stretching and turbulent eddy growth in the flow, and this leads to reduce the energy dissipation and to cause drag reduction.

The extensional viscosity characteristics of cationic surfactant solution with suitable counter-ion are more complex than that of polymer solution. Prud'homme and Warr') pointed out that extensional thickening is observed as a result of micelle growth in the middle extensional range. Walker and Moldenaers ${ }^{10)}$ reported such an extensional thickening becomes weaker for biaxial extensional flow cases. In their discussion, however, the concentration of surfactant is limited at a higher value than the usually used one in a drag-reducing pipe flow.

The characteristics of the extensional viscosity are of importance not only for the drag reduction phenomena but also for the complex geometry flow such as having a sudden 
expansion or a sudden contraction. Suzuki et al. ${ }^{11,12)}$ discussed the flow structure of a dilute surfactant solution in a cavity in order to investigate the heat transfer characteristics in a heat exchanger for a visco-elastic fluid. For this case, the extensional viscosity seriously affects the formation of the recirculation region observed in a cavity.

In this paper, the biaxial extensional characteristics of cationic surfactant solution at a dilute concentration, which is less than 1,000 ppm, are measured by using opposed-nozzle instruments. Especially, the time characteristics of micellar network formation and relaxation are focused. The time characteristics of the micellar network structure are very important because it is closely related both to the turbulence control in a drag-reducing flow and to the formation of a recirculation region in a cavity flow.

\section{EXPERIMENTS}

The drag reducing cationic surfactant, oleylbishydroxyethyl methylammonium chloride, trade name: Ethoquad $\mathrm{O} / 12$, and additive salt which ionized to counter-ion in aqueous solution, sodium salicylate, $\mathrm{NaSal}$, were used in these experiments. The concentrations of surfactants are varied from 250 to $1,000 \mathrm{ppm}$ and the molar ratio of additive salt to surfactant is kept constant at 1.5. Solvent used in these experiments was ionexchanged water. The samples were put to equilibrate for more than one day.

A Rheometrics RFX Fluid Analyzer, which used two opposed nozzles to generate an extensional flow, was used to measure the extensional viscosity and the time variation of the extensional stress worked on the fluid. Figure 1 shows a schematic view of its setup. The biaxial extensional flow mode (ex-flux mode) was employed with this instrument as shown in Fig. 2. The nozzle diameter and the distance between

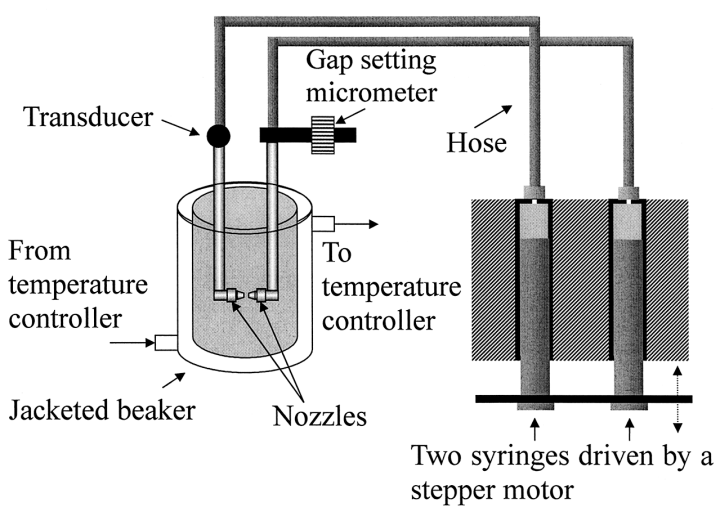

Fig. 1. The schematic diagram of experimental instrument. nozzles were set at $0.5 \mathrm{~mm}$ during the measurements because the extensional stress did not take a final plateau value with larger diameters. The temperature of surfactant solutions was controlled to $25 \pm 0.5^{\circ} \mathrm{C}$ by a temperature controller. Surfactant solution was sucked into two syringes before the beginning of the experiments and then expelled out of syringes at a constant flow rate by a stepping motor. Because the extensional stress is very sensitive with the structure of surfactant micellar network formed by self-assemble mechanism before measurements, all samples were sucked into the syringes at the same high extensional rate $\left(8,000 \mathrm{~s}^{-1}\right)$ in order to create the same initial condition for all measurements. The time variations of extensional stress signals were recorded by a logger station with the sampling time rate is set equal to $100 \mathrm{~ms}$. All experiments were carried out three times and the analysis results on the extensional viscosity were taken as the averaged value of these three measurement results.

A Rheometrics ARES with a $50 \mathrm{~mm}$ diameter cone-plate fixture is used to measure the shear viscosity and time variation of the shear stress in the shear flow. For comparison, they are measured at $25^{\circ} \mathrm{C}$. The shear viscosity and relaxation time of shear stress in shear flow are shown in Figs. 3 and 10, respectively. Because of the limit of the experimental instrument the shear flow data is measured only up to the shear rate of $1000 \mathrm{~s}^{-1}$ which corresponds to the region I of the extensional flow as shown in Fig. 6.

\section{RESULTS AND DISCUSSION}

\subsection{Extensional Viscosity and Extensional Stress}

Figure 3 shows the shear viscosity of various surfactant solutions in the shear flow. The computational results of shear viscosities by using the modified Bird-Careau model for the

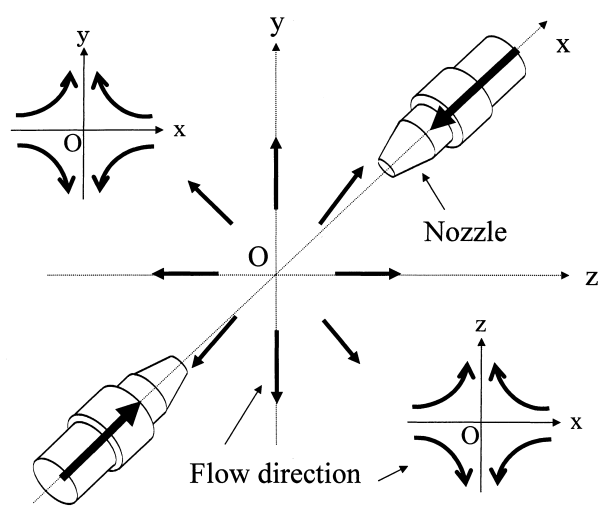

Fig. 2. The direction of biaxial extensional flow between two opposed nozzles. 
cases of $500 \mathrm{ppm}$ and 1,000 ppm are also plotted. By this method, the shear viscosity is estimated by using the following equations: ${ }^{13,14)}$

$$
\eta=\eta_{0}\left[1+\left(t^{\prime} \dot{\gamma}\right)^{2}\right]^{\frac{n-1}{2}}+\eta_{\infty}
$$

where $\eta[\mathrm{Pa} \cdot \mathrm{s}], \eta_{0}[\mathrm{~Pa} \cdot \mathrm{s}], \mu_{\infty}[\mathrm{Pa} \cdot \mathrm{s}], t^{\prime}[\mathrm{s}], n[-]$ are shear viscosity, zero shear viscosity, infinite viscosity, time constant and power index, respectively. For the case of $500 \mathrm{ppm}$, they are calculated by:

$$
\begin{aligned}
& \eta_{0}=9.33 \times 10^{-13} \exp \left(\frac{7,137}{T}\right) \\
& \eta_{\infty}=1.27 \times 10^{-3} \exp \left[2,190 \times\left(\frac{1}{T}-\frac{1}{291}\right)\right] \\
& t^{\prime}=1.86 \times 10^{-8} \exp \left(\frac{4,400}{T}\right) \\
& n=0.005
\end{aligned}
$$

For the case of $1,000 \mathrm{ppm}$, the following equations are employed:

$$
\begin{aligned}
& \eta_{0}=1.076 \times 10^{-6} \exp \left(\frac{3,137}{T}\right) \\
& \eta_{\infty}=8.01 \times 10^{-7} \exp \left(\frac{2,090}{T}\right) \\
& t^{\prime}=3.014 \times 10^{-6} \exp \left(\frac{2,771}{T}\right)
\end{aligned}
$$

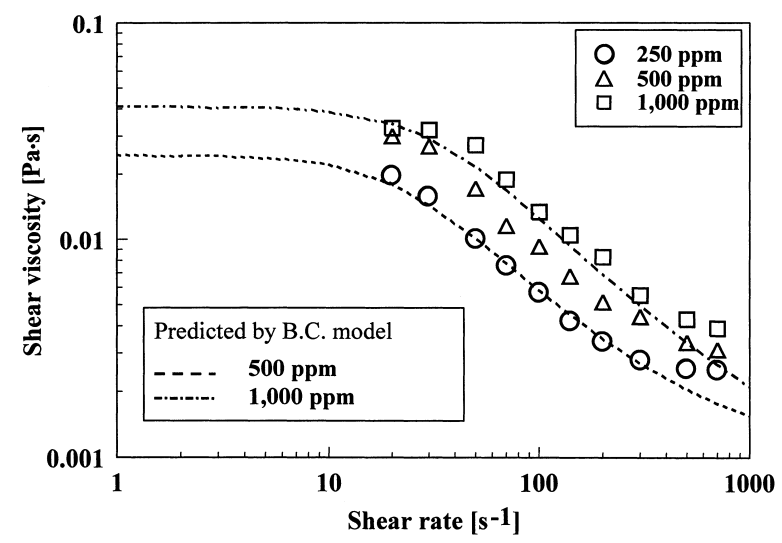

Fig. 3. Shear viscosity vs. shear rate for various surfactant concentrations measured by Rheometrics ARES.
From the figure, it is found that a shear thinning occurs at high shear rate region and the shear viscosity increases when the concentration of surfactant increases. This is caused by the deformation of shear induced state, SIS, reported by Orlendorf et $a l .{ }^{15)}$ at high shear rate.

Figure 4 shows the apparent extensional viscosity variation of the solvent and those of surfactant solutions on the extensional rate, $\dot{\varepsilon}\left[\mathrm{s}^{-1}\right]$, for various concentrations. From the figure, the apparent extensional viscosity, $\eta_{E}[\mathrm{~Pa} \cdot \mathrm{s}]$, of solvent is found to increase proportionally to the extensional rate. This result is consistent with the results that Dontula et al. ${ }^{16)}$ have measured for low extensional viscosity liquids at high extensional rate by using in-flux mode of the Rheometrics RFX. According to them, this is caused by the effect of inertia. On the other hand, the apparent extensional viscosity for each surfactant solution shows a complicated manner extremely depending on the extensional rate. In low extensional rate region, it first shows a gradual extensional thinning. This tendency is weak but it is proved by the fact that each solution shows a high Trouton ratio than that of a Newtonian, and the stress of each solution shows two relaxation processes in the shear flow and also in the extensional flow as mentioned below. At a certain extensional rate, a rather steep thickening is observed until taking a sharp peak value. After the peak, the extensional viscosity decreases again until the final extensional thickening occurs as observed in the case of the solvent.

Figure 5 shows the Trouton ratio, i.e. the ratio of extensional viscosity and zero-shear viscosity, of various surfactant solutions. The values of zero shear viscosity of these solutions are calculated from Eqs. (2) and (6) for cases of $500 \mathrm{ppm}$ and $1,000 \mathrm{ppm}$ surfactant solution. For the case of $250 \mathrm{ppm}$ solution, it is predicted from the extrapolating low shear

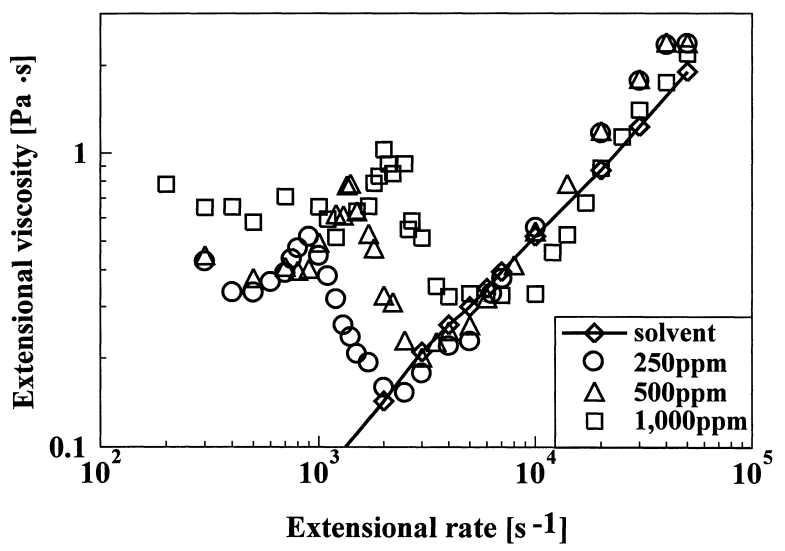

Fig. 4. Extensional viscosity vs. extensional rate for various surfactant concentrations. 
viscosity to zero shear rate. From the figure, this ratio shows a value from about 8 to 120 while for a Newtonian fluid it equals to 6 for a biaxial extensional flow. Especially, in the region I which is clarified in Fig. 6, the Trouton ratio shows a value over 14. In addition, the shear stress of each surfactant solution shows two relaxation time processes in the shear flow as shown in Fig. 10 while they are not found for a Newtonian fluid flow. From these, the extensional viscosity behavior in the region I which is clarified in Fig. 6 is not a plateau region but an extensional thinning occurrence.

The extensional viscosity behaviors of surfactant solutions are classified into four regions of extensional rate. Figure 6 shows the classification of this behavior in the case of 1,000 ppm concentration solution. The regions I and III correspond to the regions where the first and the second extensional thinning phenomena appear, respectively, while the regions II and IV correspond to the regions where the first and the second extensional thickening phenomena appear. In the regions I and III, the micellar network structures are simply broken by the extensional rate. The second extensional

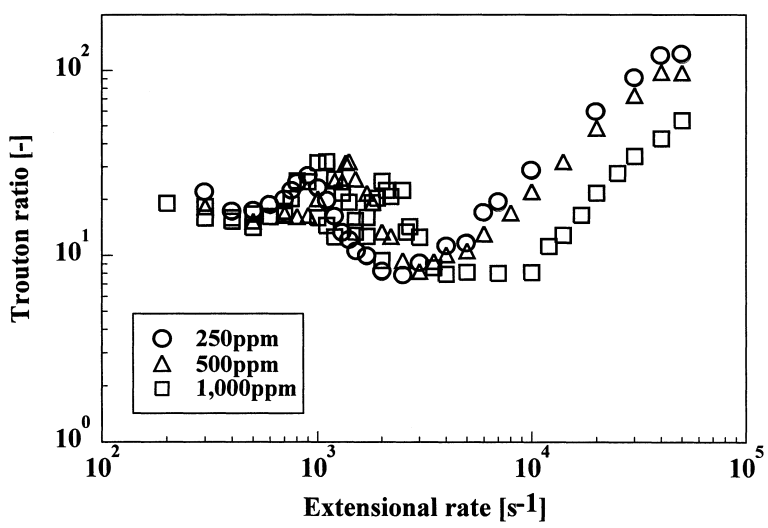

Fig. 5. Trouton ratio of various surfactant solutions.

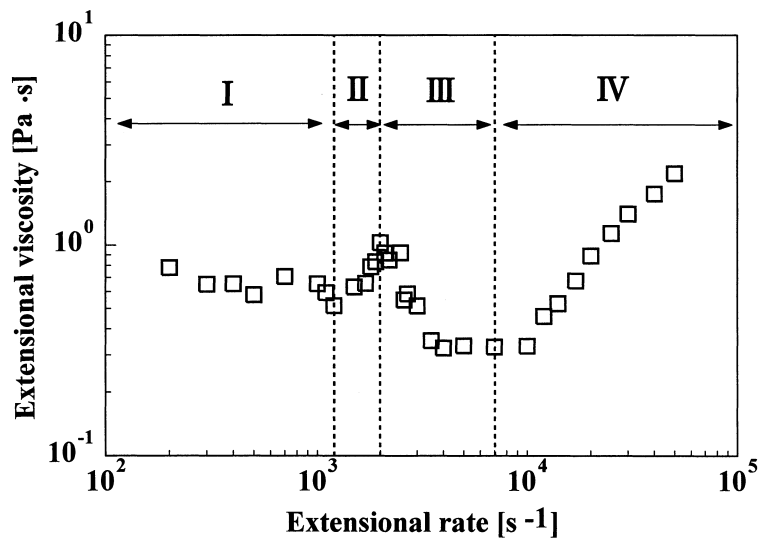

Fig. 6. Four regions of extensional rate for 1,000 ppm solution. thickening observed in the region IV is caused by the inertia as mentioned above. Prud'homme and Warr measured extensional viscosity in higher concentration than that of the present study and found the similar extensional thickening as observed in region II. According to them, the micellar structures lay in the same direction in such a region.

\subsection{Time Characteristics of Extensional Stress}

In each region, the extensional stress has its own time variation behavior approaching to the plateau value. In this section, the time characteristics of extensional stress during measurements will be discussed in detail.

Figure 7 shows the time variation of extensional stress approaching to the plateau value for each region at the concentration of $1,000 \mathrm{ppm}$. The extensional stresses at 500, $1,500,2,200$ and $10,000 \mathrm{~s}^{-1}$ of extensional rate are the examples representing the region I, II, III and IV, respectively.

From the figure, the stress is found to increase until gradually reaching a plateau in the region I. On the other hand, in the region II, the stress takes an overshooting peak value

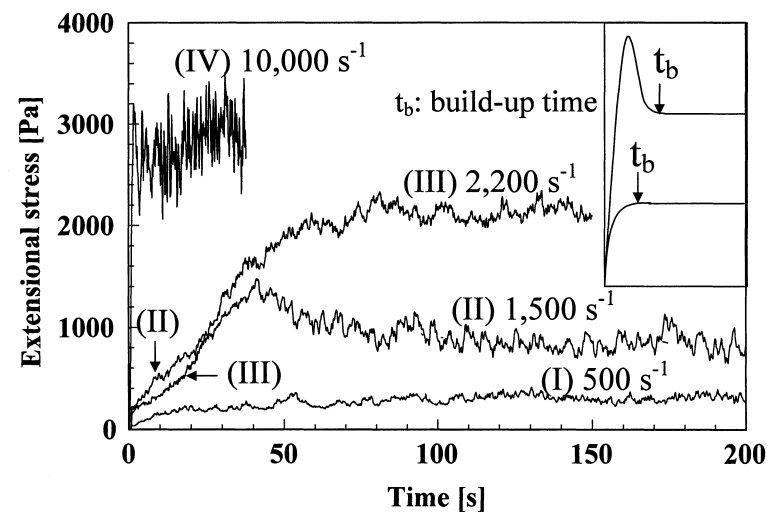

Fig. 7. Time dependency of extensional stress for $1,000 \mathrm{ppm}$ surfactant solution.

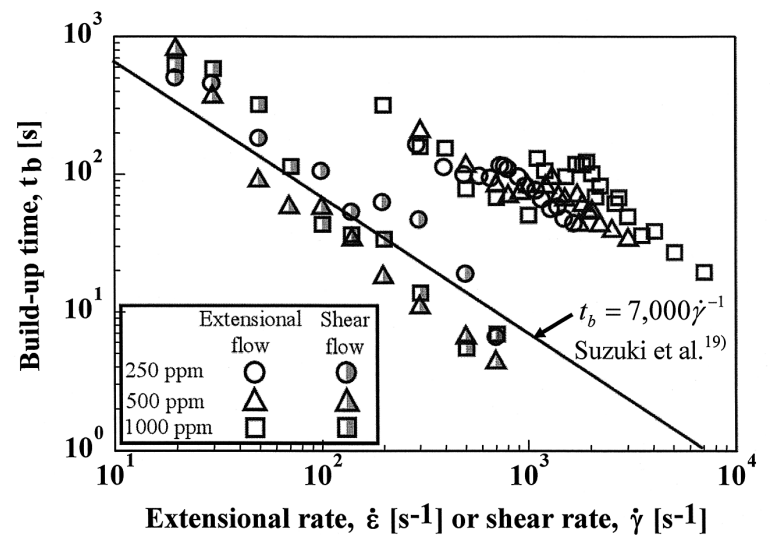

Fig. 8. Build-up time for each concentration. 
before reaching a final plateau. The stress in the region III increases gradually to reach a plateau, again. In the region IV, the stress very rapidly reaches a plateau. These characteristics are deeply related to the micellar network structure formation. In the region I, a small-scale but stable micellar network structure is formed gradually by extensional stress addition. The region II can be considered to be a kind of transit region. There, the micellar network structure is reconstructed, and then the micellar network structure becomes large as Prud'homme and Warr mentioned. This large-scale network causes extensional thickening but still has some instability or non-uniformity in the region II. On the other hand, the stable large-scale structure forms homogeneously as a final product in the region III. In the region IV, all network structures are broken by a high extensional rate. Then, the extensional viscosity takes almost the same value as that of the solvent.

Figure 8 shows the build-up time, $t_{b}[\mathrm{~s}]$, of shear stress and extensional stress at various concentrations of surfactant. The build-up time is defined by the period from the shear addition to the time taking the plateau value as illustrated in the Fig. 7. The build-up time of birefringence in shear flow reported by Suzuki et al ${ }^{14)}$ for the case of 1,000 ppm of surfactant solution at the same temperature are shown by the solid line. From the figure, the build-up time of the shear stress is found to decrease as a function having a gradient of -1 in a double logarithm plot. It shows the same level with the build-up time of the birefringence. For the case of the extensional stress, it is found that the extensional stress decreases in two stages as functions that have a gradient of -1 in a double logarithm plot. It changes from a low stage to a high stage at a certain extensional rate as a result of forming a larger structure in the extensional flow. As reported for simple shear flow cases in previous study, the build-up time is dominated by the collision mechanism of

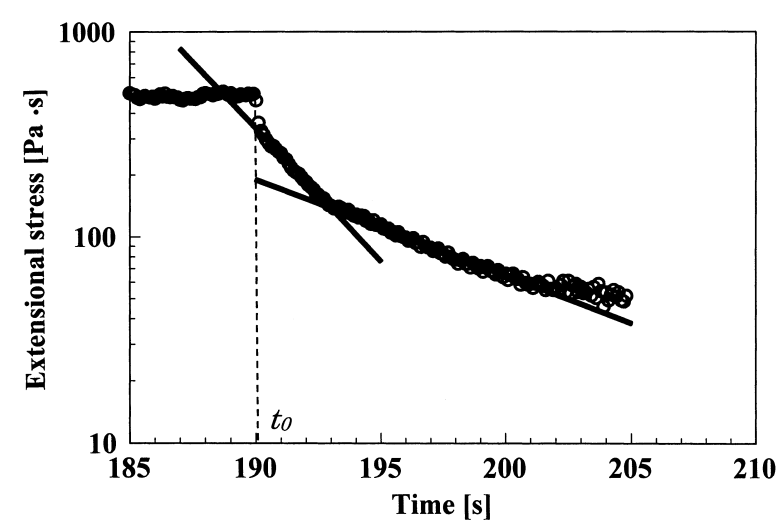

Fig. 9. Relaxation of extensional stress of $250 \mathrm{ppm}$ surfactant solution flow at extensional rate of $1,100 \mathrm{~s}^{-1}$. micellar structures. As Hu et al. ${ }^{17)}$ described, the collision rate is proportional to the shear rate for the shear flow cases. For the extensional cases, the collision rate is found to be proportional to the extensional rate. Then, the build-up time is proportional to the inverse of extensional rate. From the careful observation, it is found that the build-up time of shear stress almost does not depend on the concentration of surfactant solutions. For the case of extensional flow, the build-up time of extensional stress is found not to depend on the surfactant concentration at low extensional rate, but at high extensional rate it becomes larger as the concentration increases. Generally, the collision rate becomes larger as the concentration increases when the size of micellar structure is assumed to take the same scale independently of the concentration. This is discrepant from this fact. The present results might indicate that the final micellar structure becomes larger as the concentration increases or the number of micellar structures becomes small because an individual micellar structure becomes larger.

Figure 9 shows the relaxation behavior of extensional stress for the case of $250 \mathrm{ppm}$ surfactant solution flow after the addition of $1,100 \mathrm{~s}^{-1}$ extensional rate. Just after releasing the extensional rate, the stress decreases rapidly for about $0.4 \mathrm{~s}$. Due to the sampling limitation, the relaxation time in this region cannot be measured correctly. Then, it is not discussed in the following. Except the rapid decrease region, two relaxation times are found as shown in Fig. 9, and then the relaxation behavior is analyzed by using two relaxation times fitting as follows.

$$
\sigma_{E}=\sigma_{E 1} \exp \left(-\frac{t-t_{0}}{\tau_{1}}\right)+\sigma_{E 2} \exp \left(-\frac{t-t_{0}}{\tau_{2}}\right)
$$

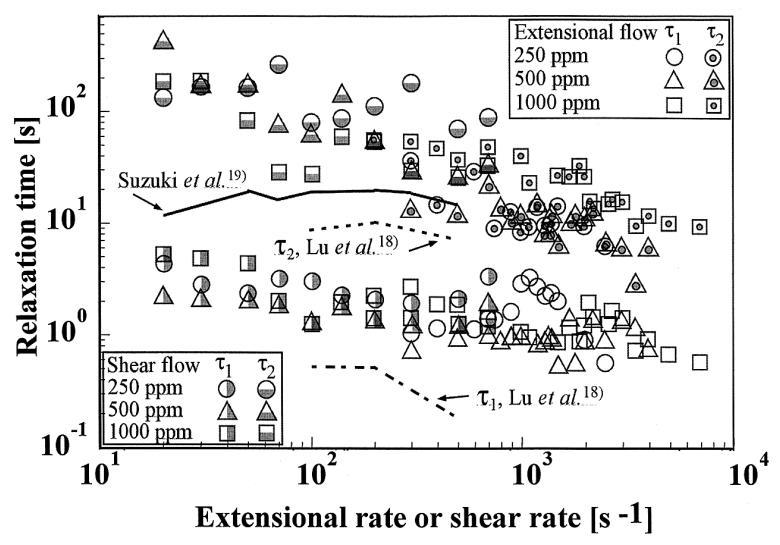

Fig. 10. Relaxation time of surfactant solutions. 
where $\sigma_{E}, \sigma_{E 1}, \sigma_{E 2}[\mathrm{~Pa}]$ are extensional stresses; $t$ [s] is measured time; $t_{0}[\mathrm{~s}]$ is the time when the extensional stress is released; $\tau_{1}, \tau_{2}[\mathrm{~s}]$ are the short and long relaxation times, respectively. Similarly, the relaxation time of the shear stress in the shear flow is calculated by this equation but extensional stresses $\sigma_{E}, \sigma_{E 1}, \sigma_{E 2}$ are replaced by shear stresses $\sigma_{S}, \sigma_{S 1}, \sigma_{S 2}$ respectively, and $t_{0}[\mathrm{~s}]$ in this case is the time when the shear stress is released. The similar analysis using two relaxation times was suggested by Lu et al. ${ }^{18)}$ for the case of shear relaxation. Figure 10 shows the short and long relaxation times of the shear stress and extensional stress for each surfactant solution calculated by using the above equation. In this figure, the relaxation time of the solution for case of 2,000 ppm reported by Lu et al. by using a cone-plate viscometry, are also plotted versus the shear rate, $\dot{\gamma}\left[\mathrm{s}^{-1}\right]$. The relaxation time of the birefringence of the same system at 1,000 ppm in the shear flow reported by Suzuki et al. ${ }^{19)}$ as a function of $t_{b}=7,000 \dot{\gamma}^{-1}$ is also shown for comparison. From the figure, it is found that the relaxation time for the extensional stress is not so strongly affected by the extensional rate and takes almost the same values as those in the shear flows cases. The latter fact indicates the structure size formed in the biaxial extensional flow is almost the same level as that formed in the shear flow. In the region II for each case of concentration, the relaxation time slightly increases with increase of the extensional rate, while it gradually decreases in the other region. This validates larger network structure of micelles forms in the region II as mentioned above.

\section{CONCLUDING REMARKS}

The extensional characteristics of a drag-reducing surfactant solution have been studied through the extensional viscosity and stress measurements in the cases of biaxial extensional flow. From the results, the extensional viscosity is found to depend on the extensional rate due to the deformation of surfactant rod-like micellar network in the extensional flow. Their behaviors are clarified into four regions that correspond to the formation and the deformation of micellar network structures in the extensional flow. In each region, their buildup and relaxation times are not strongly affected by the concentration of surfactant. On the other hand, the build-up time decreases proportionally to the extensional rate, while the relaxation time decreases weakly and affected by the extensional rate. The former fact is caused by the micellar network forms due to the collision of surfactant micelles. On the relaxation time, it is also found two relaxation processes exist for the extensional flow cases. The shorter or the longer relaxation time takes almost the same value as each value corresponding to that for the shear flow release condition. This indicates the network structure size formed in the biaxial extensional flow takes almost the same as that in the shear flow.

\section{REFERENCES}

1) Zakin JL, Christensen RN, "Reduction of Pumping Energy Losses in District Heating and Cooling Systems", Final Report, Dept. of Energy Project (1992).

2) Gasljevic K, Matthys EF, Energy and Buildings, 20, 45 (1993).

3) Usui H, Current Topics in the Physics of Fluids, 1, 121 (1994).

4) Young JCOC, Proc of 85th Int District Heating and Cooling Association Conf, p.301-317, IDHCA Pub., Tulsa, Oklahoma, USA (1992).

5) Toms BA, Proc Int Cong Rheol, 2, 135 (1949).

6) Virk PS, AIChE J, 21, 625 (1975).

7) Lu B, Li X, Zakin JL, Talmon Y, J Non-Newtonian Fluid Mech, 71, 59 (1997).

8) Bewersdoff HW, Theil H, Appl S Res, 50, 347 (1993).

9) Prud'homme RK, Warr GG, Langmuir, 10, 3419 (1994).

10) Walker L, Moldenaers P, Langmuir, 12, 6309 (1996).

11) Suzuki H, Ishiba H, Usui H, Proc 12th Heat Transfer Conference, 2, 177 (2002), Grenoble.

12) Suzuki H, Usui H, "Compact Heat Exchangers and Enhancement Technology for the Process Industries - 2003" (ed. Shah K et al.), 69 (2003).

13) Usui H, Itoh T, Saeki T, Rheol Acta, 37, 122 (1998).

14) Suzuki H, Fuller GG, Nakayama $T$, Usui H, Rheol Acta, 43, 232 (2004).

15) Orlendorf D, Interthal W, Hoffmann H, Rheol Acta, 25, 468 (1986).

16) Dontula P, Pasquali M, Scriven LE, Macosko CW, Rheol Acta, 36, 429 (1997)

17) $\mathrm{Hu} \mathrm{Y}$, Wang SQ, Jamie AM, J Colloid Interface Sci, 156, 31 (1993).

18) Lu B, Zheng Y, Davis HT, Scriven LE, Talmon Y, Zakin JL, Rheol Acta, 37, 528 (1998).

19) Suzuki H, Fuller GG, Nakayama T, Usui H, Rheol Acta, 43, 232 (2004). 